\title{
Abstract
}

\section{Teledermatology and Artificial Intelligence: Piction Health}

\author{
Susan Conover \\ Piction Health, Boston, MA, United States
}

\section{Corresponding Author:}

Susan Conover

Piction Health

55 Court St-Floor 2

Boston, MA, 02108

United States

Phone: 16179539481

Email: susan@pictionhealth.com

\section{Abstract}

Background: Skin diseases affect 2.3 billion people globally. Due to the scarcity of dermatologists, 2 in 3 cases are seen by primary care physicians (PCPs), who have lower diagnostic accuracy. Published studies have shown that the diagnostic accuracy of a PCP or general practitioner is close to $50 \%$.

Objective: The aim of this study was to build artificial intelligence (AI) classifiers across 26 and 54 common and urgent adult rashes that present in a primary care setting.

Methods: We trained our AI models with approximately 50,000 total photos. The number of images within each disease or class ranged from 76 to 5505. Additionally, we further tested narrowing the differential diagnosis by adding body part information to identify how this impacts top-5 accuracy for one condition.

Results: Overall, we trained an AI model to identify 26 classes on par with the accuracy level of a dermatologist, who is, on average, $75 \%$ top-3 accurate across 26 conditions. Additionally, we trained the AI model across 54 conditions and achieved $74.3 \%$ top-5 accuracy across common conditions and $79.2 \%$ top-5 accuracy across urgent conditions. In evaluating if body part information may increase top-5 accuracy, we saw top-5 accuracy for one condition increase from $67 \%$ to $97 \%$.

Conclusions: Overall, we concluded that including body part information to down-select possible disease matches substantially increased the overall differential diagnosis accuracy for body region-specific conditions. We also concluded that AI may assist PCPs to identify the most likely skin conditions quickly in a clinical encounter, improve overall diagnostic accuracy, and inform the most appropriate next step for the patient. These promising findings highlight the need and potential of AI and clinical decision support to augment the ability of PCPs to accurately and confidently evaluate patients with skin conditions.

Conflicts of Interest: SC is the cofounder and chief executive officer of Piction Health, a company focused on using AI to help augment frontline providers' clinical decision making to save time and improve outcomes for patients with skin diseases. SC also holds shares in Piction Health.

(iproc 2022;8(1):e36905) doi: $10.2196 / 36905$

\section{KEYWORDS}

artificial intelligence; machine learning; clinical decision support; dermatology; AI

\section{Multimedia Appendix 1}

Top-3 diagnostic accuracy of health care providers across 26 skin conditions, in comparison to Piction Health. [PNG File, 25 KB-Multimedia Appendix 1] 
Edited by T Derrick; this is a non-peer-reviewed article. Submitted 28.01.22; accepted 28.01.22; published 08.03.22.

Please cite as:

Conover $S$

Teledermatology and Artificial Intelligence: Piction Health

iproc 2022;8(1):e36905

URL: https://www.iproc.org/2022/1/e36905

doi: $\underline{10.2196 / 36905}$

PMID:

CSusan Conover. Originally published in Iproceedings (https://www.iproc.org), 08.03.2022. This is an open-access article distributed under the terms of the Creative Commons Attribution License (https://creativecommons.org/licenses/by/4.0/), which permits unrestricted use, distribution, and reproduction in any medium, provided the original work, first published in Iproceedings, is properly cited. The complete bibliographic information, a link to the original publication on https://www.iproc.org/, as well as this copyright and license information must be included. 